\title{
Spathe morphology of Commelina L. (Commelinaceae) of India in relation to Taxonomy
}

\section{Sheba M. Joseph and Santhosh Nampy*}

Plant Systematics and Floristics Lab, Department of Botany, St. Joseph's College, Devagiri, Kozhikode, Kerala-673 008, India

\begin{abstract}
Commelina L., the largest genus of Commelinaceae, is easily distinguished from related genera by inflorescence subtended in a folded bract (spathe). The position, number, size, shape, hairiness, cincinnus number and peduncle length of the spathe are useful in species delimitation. In the present investigation, twenty four species of Commelina occurring in India are studied for spathe characters and their significance in taxonomy is discussed. A key for the identification of all species based on spathe characters is also provided.
\end{abstract}

Key-words: Commelina, spathe morphology, taxonomy, India.

\section{Introduction}

Commelina L. belongs to the spiderwort family Commelinaceae and comprises about 205 species (Govaerts 2009). The genus is easily distinguished from related genera by its terminal and leaf opposed inflorescence composed of 1 or 2 cincinni enclosed in a folded spathe (Faden 1998). It is represented by 22 species in India (modified after Karthikeyan et al. 1989). Recently, two more species viz., Commelina clavatoides Nampy \& S.M. Joseph (Sheba et al. 2012) and C. andamanica S.M. Joseph \& Nampy (Nampy and Sheba 2012) are added to the Indian Flora, thus bringing the total number into twenty four.

Subsequent to Hooker's treatment of the family in Flora of India (Hooker 1892), which is based on C.B. Clarke's (1881) worldwide monograph of the family, there has been no revision of the genus Commelina in India. Most of the recent studies pertain to purely cytological

*Correspondence, e-mail: santhoshnampy@yahoo.com; Tel: 04952355901(Office), 09447461622 (Mobile); Fax- 0495-2357370. or cytotaxonomical aspects of a few species (Murthy 1934; Sharma 1955; Sharma and Sharma 1958; Kammathy and Rao 1961a, 1961b, 1964; Raghavan and Rao 1965; Shetty and Subramanyam 1962; Panigrahi and Kammathy 1962; Rao 1964, 1966; Rao et al. 1968, 1970). The other works include the treatments in regional floras by various authors, to cite a few: Wight (1853), Rama Rao (1914), Bamber (1916), Fischer (1931), Fyson (1932), Barnes (1946), Haines (1961), Maheswari (1963), Sha (1978), Sharma and Kachroo (1981), Deb (1983), Chowdhary and Wadhwa (1984), Sharma et al. (1984, 1996), Matthew (1999), Henry et al. (1989), Shetty and Singh (1991), Hajra and Verma (1996), Pullaiah (1997); Hajra and Rao (1999), Singh et al. (2001), Bora and Kumar (2003), Nayar et al. (2006). Nampy and Joby (2002-2005, unpublished) have done taxonomic studies on this family in southern peninsular India. The only other work, after Hooker (l.c.), that covers the genus for the whole of India is the recent enumeration published by Karthikeyan et al. (1989) in the Florae Indicae Enumeratio Monocotyledonae. The present work is part of an ongoing study on the revision of Commelina in India. Recently, Sheba and Nampy 
(2012) have discussed the significance of capsule and seed morphology of Indian Commelina in relation to taxonomy. In the present paper, the significance of spathe characters in relation to taxonomy is discussed.

\section{Materials and Methods}

The present study is based on the specimens collected from different parts of India and cultivated at the St. Joseph's College Botanical Garden. In addition, specimens from the following herbaria (abbreviations follow Index Herbariorum) are also consulted: ASSAM, BLAT, BSD, BSI, BSJO, CAL, CALI, DD, DEV, JCB, MH, PBL, RRLB, RHT, SKU, TBGT. Two thousand seven hundred and eighty seven herbarium specimens and four hundred and twenty three populations representing 24 Commelina species are observed in the field in India during 2008-12 (only representative specimens are cited in the text).

The spathe are carefully removed and mounted on a clean glass slide. Observations are made with a LEICA MZ 7.5 Stereo zoom microscope and for photograph a Nikon D 50 Camera is used. Position, number, size, shape, hairiness, peduncle length, number of cincinni in the spathe and the number of flowers in each cincinnus are observed. Voucher specimens are deposited at St. Joseph’s College Herbarium (DEV).

\section{Results and Discussion}

Spathe morphology of 24 Indian species of Commelina are investigated, taking into consideration the position, number, size, shape, hairiness, length of peduncle and nature of cincinni. The spathe provides many taxonomically useful characters that help in distinguishing species of Commelina.

\section{SHAPE}

Spathes are of two kinds: cucullate and conduplicate. Cucullate spathes are funnel shaped due to the fusion at the basal region. The apex is acuminate or acute. Apex is falcate in C. albescens. In conduplicate spathes, the margins are free to the base but they are folded. Among the 24 Indian species, 10 come under cucullate type, including $C$. petersii and $C$. paleata though they resemble conduplicate type in appearance. All the cucullate species possess mucilaginous secretions within the spathe.

\section{SIZE}

The size of the spathe varies from 1-9 cm long. The smallest one, about $1 \mathrm{~cm}$ long, is found in C. subulata and the longest spathe, about $9 \mathrm{~cm}$ long, is found in $C$. appendiculata.

\section{NUMBER}

The number of spathe with in a sheath is solitary or a cluster of up to 6 or 7. Among species with cucullate spathe, C. forskalaei, C. ensifolia, C. petersii and $C$. paleata bear solitary spathe while $C$. maculata $C$. paludosa, C. kurzii, C. benghalensis and C. albescens have 2-6 spathe. But all species with conduplicate spathes bear single spathe within a leaf sheath.

\section{POSITION}

The position is always terminal in cucullate type spathe, but in conduplicate type, though they are terminal, later become leaf opposed. Among the conduplicate type $C$. suffruticosa and $C$. coelestis have always terminal spathe.

\section{PUBESCENCE}

The spathe of Commelina is ciliate, pubescent, puberulent or glabrous. Among cucullate type, $C$. paludosa alone bear glabrous spathe. In all other species spathe is ciliate or glandular hairy. In conduplicate type, C. appendiculata, C. diffusa and C. longifolia are glabrous and the remaining species with short or long ciliate or puberulent. In C. subulata and $C$. wightii margin is ciliate throughout. In $C$. caroliniana and $C$. clavata, the cilia are restricted towards the base.

\section{CINCINNUS NUMBER}

The number of cincinnus is usually 2, but in some species the upper cincinnus is aborted or not exserted. Among 
species with cucullate spathe, C. forskalaei, and $C$. benghalensis alone have exserted upper cincinnus while in others they are lacking or vestigial. In species with conduplicate spathe, the upper cincinnus is generally exserted or vestigial except in C. suffruticosa, where it is completely lacking.

\section{PEDUNCLE LENGTH}

The spathe is pedunculate or sessile. Among cucullate type, C.petersii is long pedunculate and C. forskalaei is short pedunculate and others are sessile or sub sessile. Among conduplicate type, $C$. subulata is always sessile while in others the length varies from 2 to $7 \mathrm{~cm}$.

KEY TO THE INDIAN SPECIES OF COMMELINA BASED ON SPATHE CHARACTERS

1. Spathes cucullate. .2 Spathes conduplicate

. Spathes sessile or sub sessile, peduncle less than $1.5 \mathrm{~cm}$ long .................................................. Spathes distinctly pedunculate, peduncle more than 1.5 cm long .... ..C. petersii

3. Spathes axillary C. forskalaei Spathes terminal .................. 4

4. Spathes solitary Spathes clustered.

5. Spathes produced on short lateral branches Spathes produced on the main branches

C. andamanica

6. Spathes with prominent veins; peduncle up to $0.5 \mathrm{~cm}$ long .C. ensifolia Spathes without prominent veins; peduncle up to $1.5 \mathrm{~cm}$ long C. paleata

7. Spathes glabrous C. paludosa Spathes pubescent, puberulous or ciliate C. albescens

8. Spathes falcately hooked at one end Spathes not falcately hooked

9. Spathes 1 cincinnate Spathes 2 cincinnate

10. Spathes pilose-puberulous out; peduncle $0.1-0.3 \mathrm{~cm}$ long. .. C. kurzii Spathes ciliate out; peduncle absent. C. maculata

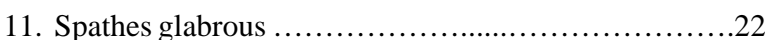
Spathes ciliate or puberulous ........................12

12. Spathes margin ciliate $\ldots 13$
Spathes margin not ciliate

13. Cilia restricted to the basal region of the spathe ...........14 Cilia throughout the margin of the spathe .................16

14. Upper cincinnus exserted .................................15 Upper cincinnus not exserted .....................C. caroliniana

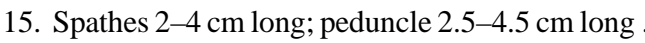
................................................... Clavata Spathes $4.5-5 \mathrm{~cm}$ long; peduncle $6-10 \mathrm{~cm}$ long... C. clavatoides

16. Spathes less than $2 \mathrm{~cm}$ long.......................... subulata Spathes more than $2 \mathrm{~cm}$ long............................ 17

17. Spathes prominently veined; 1 cincinnate........C. coelestis Spathes not prominently veined; 2 cincinnate. .18

18. Lower cincinnus 4-6 flowered; flowers blue.

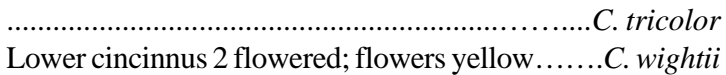

19. Spathes cordate at base ........................................... 20 Spathes hastate at base.............................. attenuata

20. Spathes acuminate at apex................... sikkimensis Spathes acute at apex..................................

21. Spathes triangular ..C. suffruticosa Spathes not triangular . C. indehiscens

22. Spathes attenuate at tip C. appendiculata Spathes not attenuate at tip 23

23. Peduncle less than $2 \mathrm{~cm}$ long C. diffusa Peduncle more than $2 \mathrm{~cm}$ long. C. longifolia

\section{Commelina albescens Hassk. Figure $\mathbf{3 e}$}

Spathe terminal, single or clustered, 1.2-1.5 (2.5) x 0.8-1 (1.5) $\mathrm{cm}$, cucullate usually strongly falcate, acuminate at apex, surfaces puberulous or hirsute-puberulous. Peduncle $0.5 \mathrm{~cm}$ long, white, strigose. Upper cincinnus lacking; lower cincinnus $0.5 \mathrm{~cm}$ long, puberulent, 2-3 bisexual flowered.

Specimens examined: Rajasthan. Dwarka, 16/10/ 1961, T.A. Rao 76568; Jaisalmar, 11/1917, coll. unknown 9191 (BLAT). Jodhpur, 10/1917, coll. unknown 9184.

\section{Commelina andamanica S.M. Joseph \& Nampy Figure 1a}

Spathe terminal, single, 9-12 × 6.5-10 mm, cucllate, ciliate on the surfaces and margin, gree. Peducle $3-5 \mathrm{~mm}$ long. Upper cincinnus lacking, lower cincinnus ca. $5 \mathrm{~mm}$ long, strigose. 2-bisexual flowered.

Specimen examined: Andaman \& Nicober Islands, South Andaman, Chidiatapu, 24/09/2010, Santhosh 
Nampy \& Manudev 3131 (DEV). Kerala. Kozhikode district, Devagiri Botanical Garden (Cultivated), 12/12/ 2011, Sheba \& Santhosh Nampy 3751, 24/02/2012, Sheba \& Santhosh Nampy 4560, 31/03/2012, Manudev \& Santhosh Nampy 5243 (DEV).

\section{Commelina appendiculata C.B. Clarke Figure $\mathbf{3 f}$}

Spathe leaf opposed, single, 5-9 x 0.7-1.2 cm, conduplicate, slightly falcate, attenuate at apex, deeply cordate at base, outer surface glabrous, inner surface sparsely pilose, green. Peduncle 2.5-7 cm long, green, strigose. Upper cincinnus $1 \mathrm{~cm}$ long, exserted, puberulent, 1 male flowered; lower cincinnus $0.5 \mathrm{~cm}$ long, glabrous, 2 bisexual flowered.

Specimen examined: Assam. Lukhikhar forest, 26/ 06/1964, A.S. Rao 39124 (ASSAM)

\section{Commelina attenuata K.D. Koenig ex Vahl Figure 1b}

Spathe leaf opposed, single, $1.2-4.5 \times 0.2-0.5 \mathrm{~cm}$, conduplicate, acute at apex, hastate at base, outer surface puberulent, inner surface pilose, green. Peduncle $0.8-2.5 \mathrm{~cm}$. long, green, strigose. Upper cincinnus $0.3 \mathrm{~cm}$ long, exserted, ciliate, 1 male flowered; lower cincinnus 0.3-0.5 cm long, glabrous, 2 or 3 bisexual flowered.

Specimens examined: Kerala. Alappuzha district, Muhamma, 30/7/02, Anusha \& Cini 5159 (DEV). Kozhikode district, Thikkodi, 30/8/02, Santhosh Nampy \& Joby 478 (DEV). Thiruvananthapuram district, Veli, 11/10/09, Sheba \& Santhosh .Nampy 2281 (DEV).

\section{Commelina benghalensis L. Figure 1c}

Spathe terminal, clustered, 0.5-1.7 x 0.5-1.2 cm, cucullate, outer surface ciliate, inner surface glabrous, green. Peduncle absent. Upper cincinnus exserted or vestigial, ciliate, 1 male flowered; lower cincinnus ca. $0.6 \mathrm{~cm}$ long, glabrous, 2 bisexual flowered.

Specimens examined: Kerala. Kollam district, Opposite Sree Narayana College, 03/11/2010, Sheba \& Santhosh Nampy 4004 (DEV). Kozhikode district, Chelari, 02/08/2010, Santhosh Nampy \& Sheba 3247; Devagiri Botanical Garden, 03/12/2010, Sheba \& Santhosh Nampy 4047 (DEV).

\section{Commelina caroliniana Walter Figure 1d}

Spathe leaf opposed, single, $1.5-2$ x $0.5 \mathrm{~cm}$, conduplicate, acuminate at apex, broadly cordate and ciliate at base, at base, serrulate at margins, outer surface pubescent, inner surface glabrous, green. Peduncle ca. $1 \mathrm{~cm}$ long, green strigose. Upper cincinnus vestigial, 0.5-1 cm long, not exserted, ciliate; lower cincinnus $0.5 \mathrm{~cm}$ long, ciliate, 1-3 bisexual flowered.

Specimens examined: Kerala. Wayanad district, Kabani River side, 30/11/2002, Joby \& Santhosh Nampy 5103; Kalloor, 08/08/2010, Sheba \& Santhosh Nampy 3250; Muthanga, 29/11/2002, Santhosh Nampy \& Joby $893(\mathrm{DEV})$.

\section{Commelina clavata C.B. Clarke Figure 1e}

Spathe leaf opposed, single, 2-4 x 0.5-0.7 cm, conduplicate, cordate and ciliate at base, acuminate to attenuate at apex, surfaces glabrous to pilose, green. Peduncle $2.5-4.5 \mathrm{~cm}$ long, green, band of hairs on one side. Upper cincinnus $1.3-2 \mathrm{~cm}$ long, exserted, ciliate, 2 male flowered; lower cincinnus $1 \mathrm{~cm}$ long, glabrous, 4-5 bisexual flowered.

Specimens examined: Kerala. Idukki district, Elaveezhappoonchira, 25/08/2002, Joby \& Santhosh Nampy 805 (DEV). Kozhikode district, Kattullamala, 29/ 07/2010, Sheba \& Santhosh Nampy 3246 (DEV). Wayanad district, Meppadi, 26/01/2011, Sheba \& Santhosh Nampy 4038 (DEV).

\section{Commelina clavatoides Nampy \& S.M. Joseph Figure 1f}

Spathe leaf opposed, single, 4-5.5 x $1.5 \mathrm{~cm}$, conduplicate, broadly cordate and ciliate at base, acute at apex, puberulent on both surfaces, lower half pale green, upper half dark green; peduncle 6-10 cm long, green, strigose. Upper cincinnus $2-2.5 \mathrm{~cm}$ long, exsert4ed, 1 or rarely 2 male flowered; lower cincinnus $1.5-2 \mathrm{~cm}$ long, glabrous, 6 or 7 bisexual flowered.

Specimen examined: Kerala. Idukki, AdimaliMunnar Road, 19/11/2010, Sheba \& Santhosh Nampy 4016; Marayoor, 21/10/2009, Santhosh Nampy 2293 (DEV). Kannur district, Paithalmala, 14/09/2002, Santhosh Nampy \& Joby Paul 829 (DEV). Kozhikode district, Devagiri College Botanical Garden (Cultivated) 

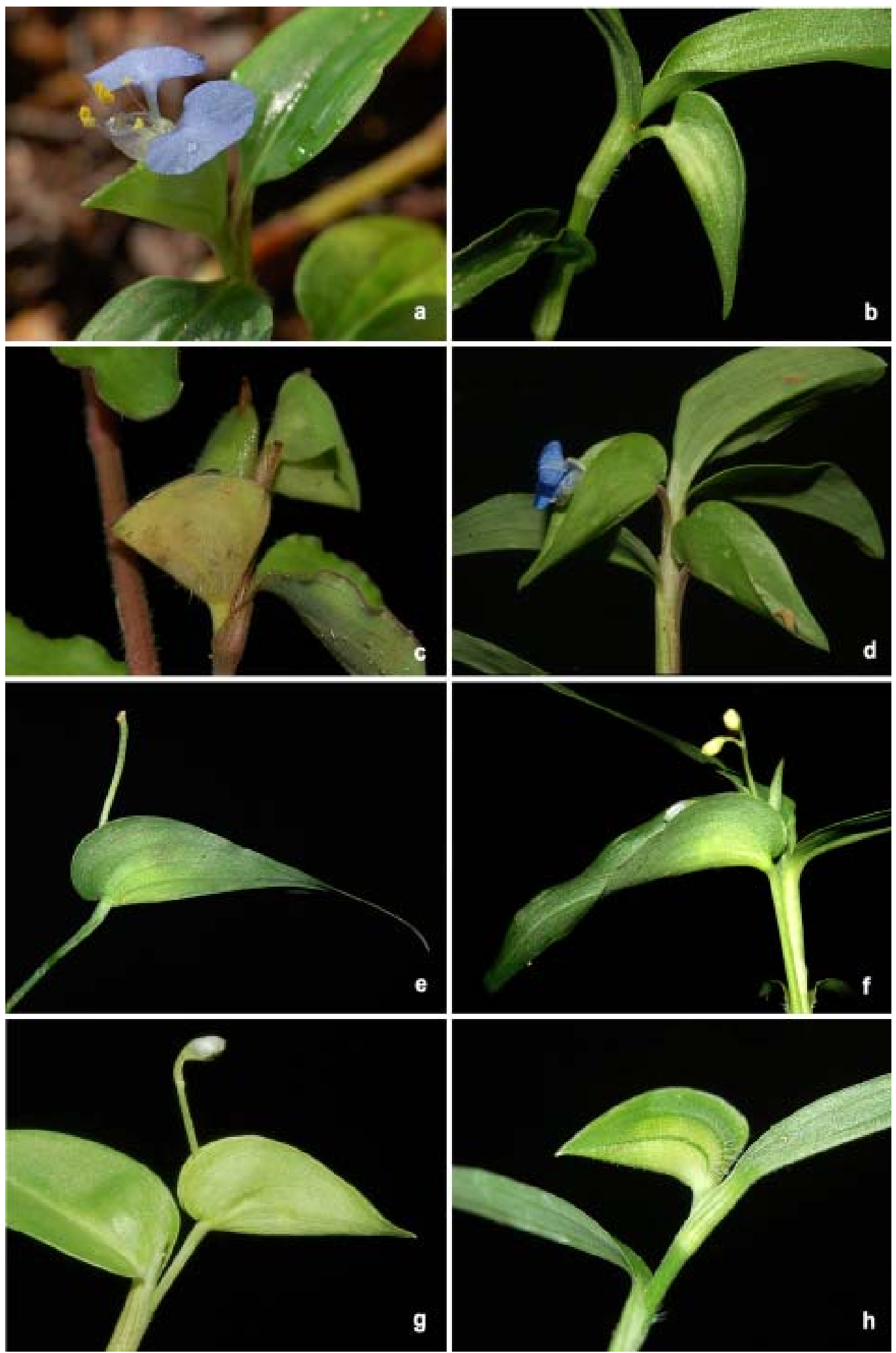

Figure 1. Spathes of Commelina species. a. C. andamanica S.M. Joseph \& Nampy; b. C. attenuata Vahl; c. C. benghalensis L.; d. C. caroliniana Walter; e. C. clavata C.B. Clarke; f. C. clavatoides Nampy \& S.M. Joseph; g. C. diffusa Burm.f.; h. C. ensifolia R. Br. 

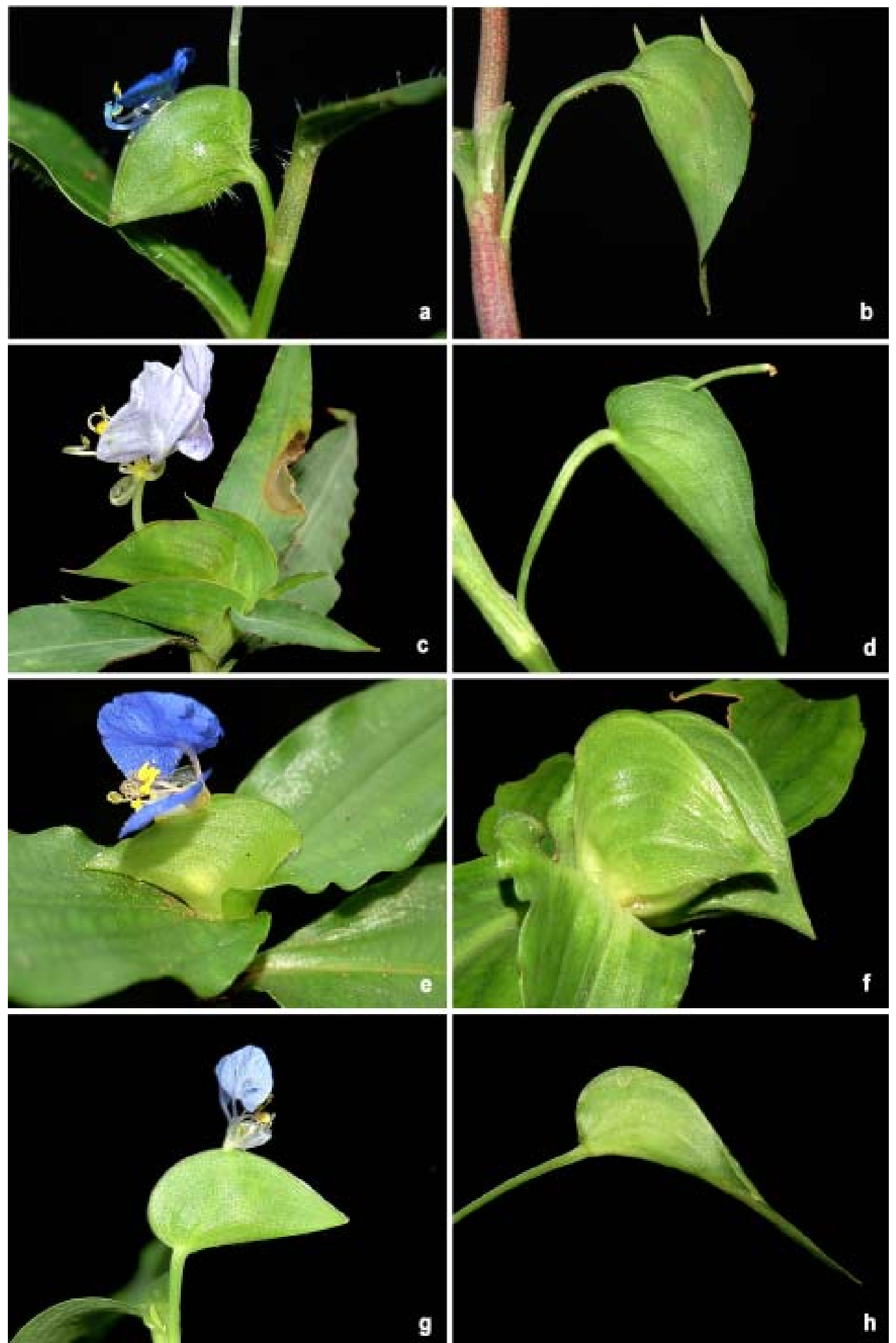

Figure 2. Spathes of Commelina species. a. C. forskalaei Vahl; b. C. indehiscens E. Barnes; c. C. kurzii C.B. Clarke; d. C. longifolia Lam.; e. C. maculata Edgew.; f. C. paludosa Blume; g. C. petersii Hassk.; h. C. sikkimensis C.B. Clarke. 
4044 (DEV). Palakkad district, Nelliyampathy, 19/11/ 2010, Shahina P.M. \& Sheba M. Joseph 4024 (DEV).

\section{Commelina coelestis Willd. Figure $\mathbf{3 g}$}

Spathe terminal in the upper leaf axils, single, $2.2-5 \mathrm{~cm}$ long, conduplicate, acuminate at apex, cordate at base, widely ovate at base, ciliate at margins, with strong longitudinal and transverse veins on both surfaces, densely hairy. Peduncle 4-8 cm long, green, pubescent. Upper cincinnus absent; lower cincinnus $0.7 \mathrm{~cm}$ long, ciliate, 8 flowered.

Specimens examined: Sikkim. -/1877, G.King s.n (MH); 13/08/1875, G.King 2239 (CAL).

\section{Commelina diffusa Burm.f Figure $\mathbf{1 g}$}

Spathe leaf opposed, single, ca. 3.5 x $1 \mathrm{~cm}$, conduplicate, acute at apex, cordate at base, glabrous on both surfaces, green. Peduncle ca. $1.5 \mathrm{~cm}$ long, green, strigose. Upper cincinnus $1.5 \mathrm{~cm}$ long, exserted, ciliate, 1 or 2 male flowered; lower cincinnus $1 \mathrm{~cm}$ long, glabrous, 2-5 bisexual flowered.

Specimens examined: Kerala. Eranakulam district, Kaloor, 12/10/2010, Sheba \& Santhosh Nampy 2299 (DEV). Idukki district, Kaliyar, 27/10/2001, Santhosh Nampy 154 (DEV). Kannur district, Madaippara, 28/09/ 2010, Sheba \& Santhosh Nampy 3495 (DEV).

\section{Commelina ensifolia R. Br. Figure $\mathbf{1 h}$}

Spathe terminal, solitary, triangular, $1-1.8 \times 0.5-5 \mathrm{~cm}$, cucullate, acute at apex, margins longly fused basally, surfaces hirsute - puberulous, pale green with darker green veins. Peduncle $0.5 \mathrm{~cm}$ long, green, pubescent. Upper cincinnus absent, lower cincinnus $0.6 \mathrm{~cm}$ long, glabrous, 2-3 flowered.

Specimens examined: Kerala. Kollam district, Thenmalai, 10/09/2003, Joby \& Santhosh Nampy 954 (DEV). Kozhikode district, Devagiri Botanical Garden, 26/07/2004, Joby \& Santhosh Nampy 1227 04/06/2010, Sheba \& Santhosh Nampy 3234(DEV).

\section{Commelina forskalaei Vahl Figure 2a}

Spathe leaf opposed, solitary, 1.5 x $0.7 \mathrm{~cm}$, cucullate, pubescent with long hairs towards the base. Peduncles
$0.6 \mathrm{~cm}$ long, green, strigose. Upper cincinnus $1 \mathrm{~cm}$ long, exserted, ciliate, 1 or 2 male flowered; lower cincinnus $0.5 \mathrm{~cm}$ long, ciliate, 4-5 bisexual flowered.

Specimens examined: Karnataka. Mysore, Zoo, 04/ 10/2001, Santhosh Nampy 138 (DEV). Kerala. Kozhikode district, Nandy beach, 03/08/2002, Joby \& Santhosh Nampy 480 (DEV); Kappadu beach, 28/10/ 2010, Sheba \& Santhosh Nampy 3297 (DEV).

\section{Commelina indehiscens E. Barnes Figure $\mathbf{2 b}$}

Spathe leaf opposed, solitary, 2-3 x 1-1.2 cm, conduplicate, acuminate at apex, broadly cordate at base, outer surface pilose, inner surface puberulous, green. Peduncle $2.5-3 \mathrm{~cm}$ long, green, strigose. Upper cincinnus usually lacking or occasionally present, 1-1.2 cm long, exserted, puberulous, 1-2 male flowered; lower cincinnus $1 \mathrm{~cm}$ long, glabrous, 3-6 bisexual flowered.

Specimens examined: Kerala. Idukki district, Poopara-Munnar roadside, 27/12/2001, Santhosh Nampy 438; Thodupuzha, 16/10/ 2010, Sheba \& Santhosh Nampy 3830 (DEV). Wayanad district, Meppadi, 26/01/ 2011, Sheba \& Santhosh Nampy 4037 (DEV).

\section{Commelina kurzii C.B. Clarke Figure 2c}

Spathe terminal, clustered, 2.5-3 x 1-1.5 cm, cucullate, acute at apex, basally fused, outer surface pilosepuberulous, inner surface glabrous; green. Peduncle 0.1$0.3 \mathrm{~cm}$ long, green, strigose. Upper cincinnus lacking; lower cincinnus ca. $1 \mathrm{~cm}$ long, pubescent, 2-5 bisexual flowered.

Specimens examined: Kerala. Malappuram district, Chelari, 10/06/2009, Santhosh .Nampy \& Sheba 2223 (DEV). Kozhikode district, Devagiri Botanical Garden, 27/11/2003, Joby \& Santhosh Nampy 1235, 11/12/2010, Sheba \& Santhosh .Nampy 4051(DEV).

\section{Commelina longifolia Lam. Figure 2d}

Spathe leaf opposed, single, 2.5-3 x 0.5-1 cm, conduplicate, acute at apex, cordate at base, sparsely hairy or glabrous, green. Peduncle $2-2.5 \mathrm{~cm}$ long, green. Upper cincinnus $1 \mathrm{~cm}$ long, exserted, densely ciliate, 1 male flowered; lower cincinnus $0.5-0.7 \mathrm{~cm}$ long, sparsely ciliate, 3-4 bisexual flowered. 
Specimens examined: Kerala. Alappuzha district, Kuppappuram, 20/11/2010, Sheba \& Santhosh Nampy 4018 (DEV). Wayanad district, Kalloor, 20/06/2009, Sheba \& Santhosh Nampy 3204 (DEV). Maharashtra. Pune, Nigdi, Old Bombay Highway, 03/09/2010, Sheba \& Santhosh Nampy 3264 (DEV).

\section{Commelina maculata Edgew. Figure 2e}

Spathe terminal, solitary or clustered, $0.2-1.5 \mathrm{~cm}$ long, cucullate, acute, basally fused, ciliate on the outer surface, glabrous on the inner surface, green. Peduncle absent. Upper cincinnus absent; lower cincinnus ca. $0.5 \mathrm{~cm}$ long, ciliate, 3 bisexual flowered.

Specimens examined: Kerala. Wayanad district, Muthanga Wild life Sanctuary, 27/09/09, Sheba \& Santhosh Nampy 2277 (DEV). Tamil Nadu. Kodaikanal, Palani road, 21/10/2009, Sheba \& Santhosh Nampy 3300 (DEV). West Bengal. Darjeeling, Kurseong, 29/08/2009, Sheba \& Santhosh Nampy 2235(DEV).

\section{Commelina paleata Hassk. Figure $\mathbf{3 h}$}

Spathe terminal, single, $1.8-3.2$ x $1.5 \mathrm{~cm}$, cucullate, acuteacuminate at apex, basally fused, broadly rhomboid at base, outer surface pubescent, inner surface glabrous. Peduncle 0.9-1.5 cm long, strigose, green. Upper cincinnus lacking or 1or 2 male flowered; lower cincinnus 0.5-0.6 cm long, glabrous, 4 bisexual flowered.

Specimens examined: Maharashtra. Khandala, 06/ 08/1944, Santapau 4656; Vicinity of C. Diary, 07/07/1958, S.C.Tavakary 1280; 07/1919, Santapau 27396 (BLAT); Kanheri Caves, 11/1962, R.S.Rao 32737; Shivneri Hills, 26/07/1963, M.Y.Ansari 88745; Matheran, 28/07/1966, B.M.Wadhwa 109816 (BSI).

\section{Commelina paludosa Blume Figure $2 \mathbf{f}$}

Spathe terminal, clustered, 2-3 x 2-2.2 cm, cucullate, acuminate at apex, basally fused, outer and inner surfaces glabrous, green. Peduncle $0.5 \mathrm{~cm}$ long, green. Upper cincinnus lacking, lower cincinnus $1 \mathrm{~cm}$ long, glabrous, 5 or 6 bisexual flowered.

Specimens examined: Kerala. Kannur district, Kottiyoor forest, 01/10/2003, Joby \& Santhosh Nampy
998 (DEV). Kozhikode district, Adivarum-Ghat road, 13/ 12/2009, Sheba \& Santhosh Nampy 2297; Devagiri Botanical Garden, 03/01/2011, Sheba \& Santhosh Nampy 4035 (DEV).

\section{Commelina petersii Hassk. Figure $\mathbf{2 g}$}

Spathe leaf opposed, single, 2-2.5 x 0.8-1 cm, margins fused basally, acute at apex, cordate - truncate at base, outer surface sticky with dense hooked hairs, inner surface glabrous, green. Peduncle 2-2.5 cm long, green, strigose. Upper cincinnus ca. $0.5 \mathrm{~cm}$ long, vestigial, represented by a short stalk, enclosed within the spathe; lower cincinnus $1 \mathrm{~cm}$ long, glabrous, 2 or 3 bisexual flowered.

Specimens examined: Kerala. Kozhikode district, Devagiri Botanical Garden, 07/06/2009, Santhosh Nampy \& Sheba 3201 (DEV).Thiruvananthapuram district, Kovalam, 19/10/2009, Sheba \& Santhosh Nampy 2284 (DEV). Tamil Nadu. Coimbatore, 27/11/2010, Sheba \& Santhosh Nampy 3973 (DEV).

\section{Commelina sikkimensis C.B. Clarke Figure $\mathbf{2 h}$}

Spathe leaf opposed, single, 2.5-4 x 0.5-0.9 cm, conduplicate, acuminate at apex, cordate at base, puberulent on both surfaces. Peduncle $2.5 \mathrm{~cm}$ long, green, strigose. Upper cincinnus 1-1.2 cm long, exserted, sparsely ciliate, 2-4 male flowered; lower cincinnus 0.7$0.9 \mathrm{~cm}$ long, glabrous, 5-6 bisexual flowered.

Specimens examined: Kerala, Kozhikode district, Devagiri Botanical Garden, (under cultivation), 04/02/ 2011, Sheba \& Santhosh Nampy 4052 (DEV). Meghalaya. Shillong, Way to Elephant Fall, 09/09/2009, Sheba \& Santhosh Nampy 2276; Moplong, 08/09/2009, Sheba \& Santhosh Nampy 2268 (DEV).

\section{Commelina subulata Roth Figure $\mathbf{3 a}$}

Spathe leaf opposed, single, ca. 1 x $0.5 \mathrm{~cm}$ long, conduplicate, acute at apex, cordate at base, ciliate along margins, outer surface puberulent, inner surface glabrous, green. Peduncle 2-3 mm long, green, strigose. Upper cincinnus absent; lower cincinnus $0.3 \mathrm{~cm}$ long, glabrous, 2-3 bisexual flowered. 

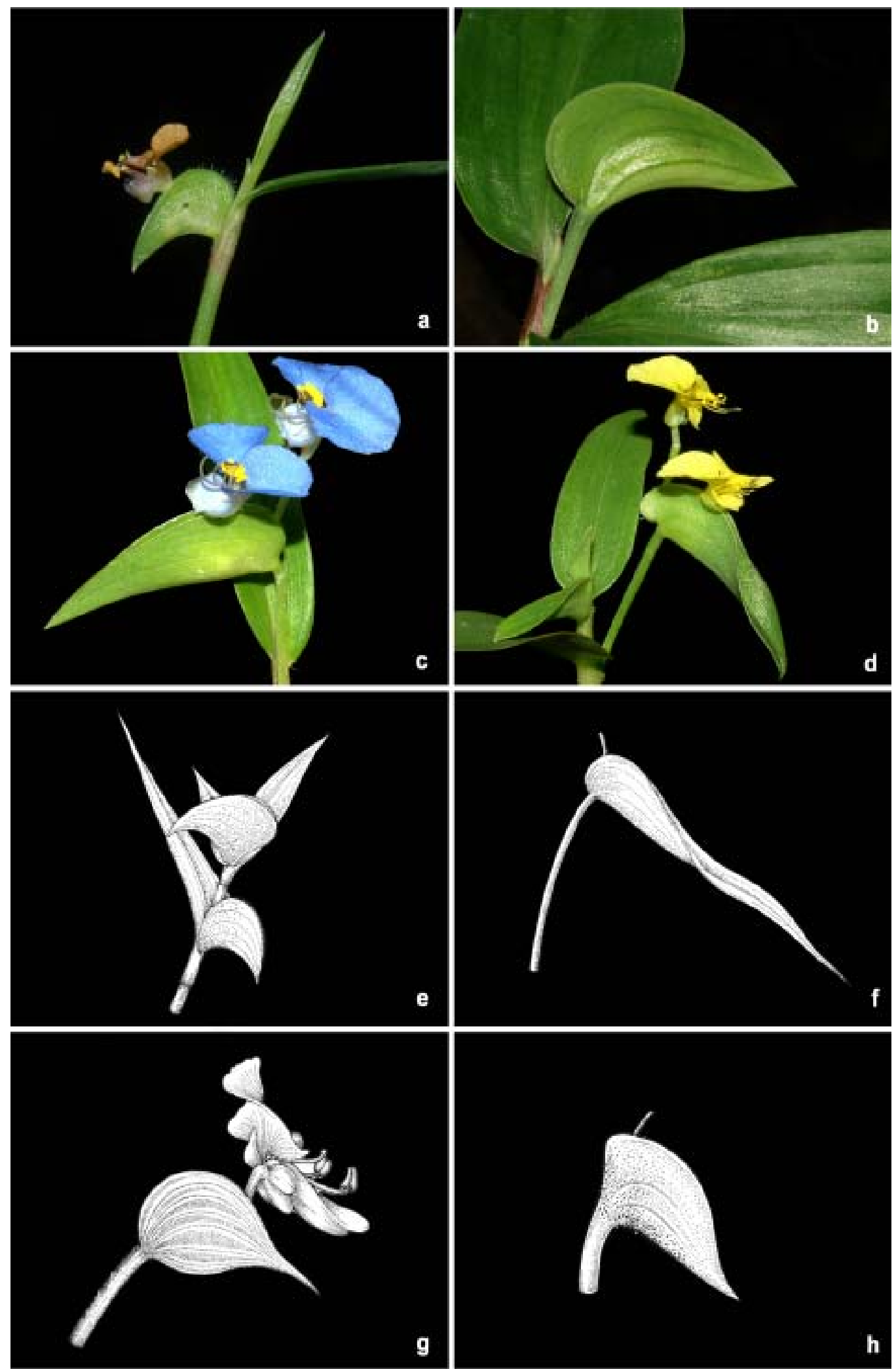

Figure 3. Spathes of Commelina species. a. Commelina subulata Roth; b. C. suffruticosa Blume; c. C. tricolor E. Barnes; d. C. wightii R.S. Rao.; e. C. albescens Hassk.; f. C. appendiculata C.B. Clarke; g. C. coelestis Wild.; h. C. paleata Hassk. (e-h drawn from herbarium specimens). 
Specimen examined: Andhra Pradesh. Chittoor district, Horsely Hill, Joby \& Ratheesh 1152 (DEV).

\section{Commelina suffruticosa Blume Figure $\mathbf{3 b}$}

Spathe terminal and axillary, solitary or clustered, 1.5-2 x $0.5-1.6 \mathrm{~cm}$, triangular, acute at apex, broadly cordate at base, outer surface pubescent, inner surface glabrous, green. Peduncle $1 \mathrm{~cm}$ long, pale green, puberulous. Upper cincinnus absent; lower cincinnus $5 \mathrm{~mm}$ long, puberulous, 6 or 7 bisexual flowered.

Specimens examined: Kerala. Kozhikode district, Devagiri Botanical Garden, 27/01/2010, Sheba \& Santhosh Nampy 4042 (DEV). Malappuram district, Adynpara waterfalls, Nilambur, 19/09/2004, Joby \& Binu 1081 (DEV). Thrissur district, Vazhani forest, 15/08/ 2003, Aneesha \& Joby 937 (DEV).

\section{Commelina tricolor E. Barnes Figure 3c}

Spathe leaf opposed,single, 3-4 x 0.8-1.2 cm, conduplicate, acute at apex, cordate at base,outer surface puberulent, inner surface glabrous, finely ciliate at margins, and green. Peduncle 4-5 cm long, green or red with row of strigose hairs. Upper cincinnus $1.5 \mathrm{~cm}$ long, exserted, glabrous, 1 male flowered; lower cincinnus 1 cm long, glabrous, 4-6 bisexual flowered.

Specimens examined: Tamil Nadu. Nilgiri district, Needle point, 06/10/2002, Nisha \& Dineshraj 847; Needle point, 26/09/2010, Sheba \& Santhosh Nampy 3485, 04/12/2010, Sheba \& Manu 4152 (DEV).

\section{Commelina wightii R.S. Rao Figure 3d}

Spathe leaf opposed, single, 2-2.5 x 0.5-0.7 cm, conduplicate, acute at apex, deeply cordate at base, ciliate at base and margins, both surfaces glabrous, green. Peduncle 2-4 cm long, green, strigose. Upper cincinnus $1 \mathrm{~cm}$ long, exserted, ciliate, 1 male flowered; lower cincinnus $0.5-0.7 \mathrm{~cm}$ long, glabrous, 2 bisexual flowered.

Specimens examined: Kerala. Kozhikode district, Devagiri botanical garden, 27/10/2010, Sheba \& Santhosh Nampy 3292 (DEV). Thrissur district, Kaveedu, 09/08/2010, Shahina \& Sheba 3254; Peruvallur, 20/09/2001, Aneesha \& Sajini 554(DEV).

\section{Acknowledgements}

The authors are grateful to Dr. C.J. Mani, Head, Department of Botany and Principal, St. Joseph's College, Devagiri, Kozhikode-8 for facilities and Mr. Manudev K.M. for photographs and drawings. One of us (SMJ) is thankful to UGC, New Delhi for awarding FIP and Principal, St. Mary’s College, Sulthan Bathery for permitting to take up the Teacher Fellowship.

\section{References}

Bamber C.J. 1916. Plants of Punjab. Government Press, Punjab, India.

Barnes E. 1946. Some observations on South Indian Commelinas: 2 new species of Commelina from South India. Journal of Bombay Natural History Society, 46: 70-89.

Bora P.J. and Kumar Y. 2003. Floristic Diversity of Assam. Daya Publishing House, Delhi, India.

Chowdhary H.J. and Wadhwa B.M. 1984. Flora of Himachal Pradesh - Vol. 3. Botanical Survey of India, Calcutta, India.

Clarke C.B. 1881. Commelinaceae. In: Monographiae Phanerogamarum - Vol. 3 (A.L.P. \& C. de Candolle, eds.), pp. 113324. G. Masson, Paris, France.

Deb D.B. 1983. Flora of Tripura State - Vol. 2. Today and Tomorrows Printers \& Publishers, New Delhi, India.

Faden R.B. 1998. Commelinaceae. In: The Families and Genera of Vascular Plants IV - Floweing Plants. Monocotyledons (Kubitzki, K., ed.). Springer Verlag, Berlin.

Fischer C.E.C. 1931. Commelinaceae. In: Flora of Presidency of Madras (J.S. Gamble, ed.), pp. 1533-1552. Adlard and Son. Ltd.

Fyson P.F. 1932. Flora of South Indian Hill Stations. Today and Tomorrows Printers and Publishers, New Delhi, India.

Govaerts R. 2009. World Checklist of Selected Plant Families. [online] URL: http://apps.kew.org/wcsp/

Haines H.H. 1961. The Botany of Bihar and Orissa - Vol. 3. Bishen Singh Mahendra Pal Singh, Dehra Dun, India.

Hajra P.K. and Rao P.S.N. 1999. Flora of Great Nicobar Island. Botanical Survey of India, Calcutta, India.

Hajra P.K. and Verma D.M. 1996. Flora of Sikkim. Botanical Survey of India, Calcutta, India.

Henry A.N., Chitra V. and Balakrishnan N.P. 1989. Flora of Tamil Nadu - Vol. 3. Botanical Survey of India, Calcutta, India.

Hooker J.D. 1892. Flora of British India. Reeve and Co. Ltd, London, UK.

Kammathy R.V. and Rao R.S. 1961a. Notes on Indian Commelinaceae II: cytological observation. Bulletin of Botanical Survey of India, 3: 167-169.

Kammathy R.V. and Rao R.S. 1961b. Notes on Indian Commelinaceae III: cytological observations. Bulletin of Botanical Survey of India, 3: 393-394.

Kammathy R.V. and Rao R.S. 1964. Notes on Indian Commelinaceae IV: cytological observation. Bulletin of Botanical Survey of India, 6: 1-6. 
Karthikeyan S., Jain S.K., Nayar M.P. and Sanjappa M. 1989. Florae Indicae Enumeratio: Monocotyledonae. Botanical Survey of India, Calcutta, India.

Maheswari J.K. 1963. Flora of Delhi. Council of Scientific and Industrial Research, New Delhi, India.

Matthew K.M. 1999. Flora of Palni Hills. Rapinat Herbarium, St. Joseph's College, Tiruchirappally, India.

Murthy K.L. 1934. Gametogenesis and Embryogeny in some Commelinaceae. Current Science, 3: 258-259.

Nampy S. and Sheba M.J. 2012. The genus Commelina (Commelinaceae) in Andaman and Nicobar Islands, India with one new species and three records. Phytotaxa (under review).

Nayar T.S., Rasiya Beegum A., Mohanan N. and Rajkumar G. 2006. Flowering Plants of Kerala. Tropical Botanical Garden Research Institute, Palode, Thiruvananthapuram, India.

Panigrahi G. and Kammathy R.V. 1962. Cytotaxonomic studies in certain species of Commelina Lnn. in Eastern India. Journal of Indian Botanical Society, 43: 294-311.

Pullaiah T. 1997. Flora of Andhra Pradesh - Vol. 1. Scientific Publisher, Jodhpur, India.

Raghavan S.R. and Rao R.S. 1965. Notes on Indian Commelinaceae VI: cytological observations. Nucleus, 8: 39-44.

Rama Rao M. 1914. Flowering Plants of Travancore. Government Press, Thiruvananthapuram, India.

Rao R.S. 1964. Indian species of Commelinaceae: miscellaneous notes. Notes Royal Botanic Garden Edinburgh, 25: 179-189.

Rao R.S. 1966. Indian species of Commelinaceae: miscellaneous notes II. Blumea, 14: 345-354.

Rao R.S., Kammathy R.V. and Raghavan R.S. 1968. Cytotaxonomic studies on Indian Commelinaceae, a review. Journal of Linnean Society of Botany, 60: 357-372.

Rao R.S., Raghavan R.S. and Kammathy R.V. 1970. Biosystematic studies on Indian Commelinaceae: the chromosome pattern and evolutionary trends. Bulletin of Botanical Survey of India, 12: $242-254$.
Sha G.L. 1978. Flora of Gujarat State. Sardar Patel University, Vallabh Nagar, India.

Sharma A.K 1955. Cytology of some of the members of the Commelinaceae and its bearing on the interpretation of phylogeny. Journal of Genetics, 27: 323-363.

Sharma A.K. and Sharma A. 1958. Further investigation on cytology of members of Commelinaceae with special reference to the role of polyploidy and the origin of the ecotypes. Journal of Genetics, 56: 1-22.

Sharma B.D., Karthikeyan S. and Singh N.P. 1996. Flora of Maharashtra (Monocotyledons). Botanical Survey of India, Calcutta, India.

Sharma B.D., Singh N.P., Raghavan R.S. and Deshpande U.R. 1984. Flora of Karnataka. Botanical Survey of India, Calcutta, India.

Sharma B.M and Kachroo P. 1981. Flora of Jammu and Plants of Neighbourhood. Bishen Singh Mahendrapal Singh, Dehra Dun, India.

Sheba M.J, Nampy S. and Anna Ancy Antony A. 2012. Commelina clavatoides (Commelinaceae): a new species from Kerala, India. Journal of Botanical Research Institute, Texas, 6: 119-122.

Sheba M.J. and Nampy S. 2012. Capsule and seed morphology of Commelina L. (Commelinaceae) in relation to taxonomy. International Journal of Botany, 8: 1-12.

Shetty B.V. and Singh V. 1991. Flora of Rajasthan - Vol. 2. Botanical Survey of India, Calcutta, India.

Shetty B.V. and Subramanyam K. 1962. Cytological studies in Commelinaceae. Nucleus, 5: 39-50.

Singh N.P., Mudgal V., Khanna K.K., Srivastava S.C., Sahoo A.K., Bandopadhyay S., Aziz N., Das M., Bhattacharya R.P. and Hajra P.K. 2001. Flora of Bihar. Botanical Survey of India, Calcutta, India.

Wight R. 1853. Icones Plantarum Indiae Orientalis (Figures of Indian Plants) - Vol. 6. Messrs Franck \& Co., Madras, India. 